\title{
¿Es Importante la Salud Oral en los Niños con Diabetes?
}

\author{
LEONOR PALOMER R. ${ }^{1}$, HERNÁN GARCÍA B. ${ }^{2}$ \\ 1. Cirujano Dentista. Profesor Asistente Adjunto de la Carrera de Odontología, Facultad de Medicina, \\ Pontificia Universidad Católica de Chile. \\ 2. Médico Endocrinólogo Infantil. Profesor Asociado Adjunto de Pediatría, Facultad de Medicina, \\ Pontificia Universidad Católica de Chile.
}

\begin{abstract}
Is Oral Health in Diabetic Children Important?

Introduction: Diabetes Mellitus (DM) affects a high proportion of the population, seen more frequently among children and adolescents. Various organs are affected, including oral tissues. Objectives: Review the relationship between DM and oral health among pediatric patients, highlighting measures that assist in prevention and early detection of these complications. Methods: A review of the literature was performed, focusing on the pediatric population. Results: Most studies agree in finding that patients with DM1 show accelerated dental exchange, gingival and periodontal disease, and abnormalities in the composition of saliva. Most studies find risk of decay, especially if there is poor metabolic control. Conclusions: Due to the increased risk, dental control should be incorporated in the multidisciplinary routine control of children with CM1. Oral health education in this risk group could decrease risk in these pathologies.
\end{abstract}

(Key words: Diabetes, diabetes mellitus, childhood diabetes, oral health).

Rev Chil Pediatr 2010; 81 (1): 64-70

\section{RESUMEN}

Introducción: La Diabetes Mellitus (DM) afecta a un porcentaje importante de la población y cada vez se observa con mayor frecuencia en niños y adolescentes. Diferentes órganos y tejidos se ven afectados en el paciente diabético, incluyendo complicaciones a nivel de los tejidos orales. Objetivo: Revisar la potencial relación entre DM y salud oral en pacientes pediátricos y buscar cuáles serían las medidas que ayudarían en la prevención y detección precoz de estas complicaciones. Método: Se realizó una búsqueda bibliográfica en Internet, ingresando a través del sitio del Sistema de Bibliotecas de la Pontificia Universidad Católica de Chile (SIBUC) a un metabuscador que permite acceder a revistas electrónicas, bases de datos y un catálogo en línea que contiene libros y revistas impresas. Se utilizaron los términos: Diabetes, Diabetes Mellitus, Diabetes Juvenil y Salud Oral. Se seleccionaron aquellos trabajos que estudiaron pacientes pediátricos. Resultados: La mayoría de los estudios concuerda en que los pacientes con DM-1 tienen recambio dental acelerado, más enfermedad gingival y periodontal, y alteración en la composición salival. Respecto de la relación con caries,

Trabajo recibido el 13 de agosto de 2009, aceptado para publicación el 07 de septiembre de 2009.

Correspondencia a:

Dra. Leonor Palomer R.

E-mail: Ipalomer@uc.cl 
la mayoría encuentra mayor riesgo de caries, especialmente en los con mal control metabólico. Conclusiones: Dado el mayor riesgo de enfermedades de la salud oral en niños con DM-1, debiera incorporarse el control dental al control multidisciplinario habitual en niños con DM-1. La incorporación de educación en salud oral es este grupo de riesgo permitiría disminuir estas patologías.

(Palabras clave: Diabetes, Diabetes Mellitus, Diabetes Juvenil, Salud Oral).

Rev Chil Pediatr 2010; 81 (1): 64-70

\section{Introducción}

La Diabetes Mellitus (DM) es una enfermedad metabólica crónica que puede comenzar durante la infancia, en la juventud o en edades más tardías. El ser portador de esta patología puede determinar que se desarrollen diferentes enfermedades que compliquen el cuadro clínico y afecten la vida de quienes las padecen. Dentro de estas, se han descrito patologías que afectan la salud oral del paciente diabético y su presentación clínica ${ }^{1}$. Dado que la severidad de las patologías orales y sus complicaciones están en gran parte determinadas por el momento que se hace el diagnóstico, es de interés conocer la fisiopatología de estas complicaciones, su frecuencia y sus manifestaciones clínicas, para así poder prevenirlas y diagnosticarlas precozmente. En esta revisión nos centraremos en las patologías orales que pueden afectar a los niños y adolescentes diabéticos.

La Diabetes Mellitus que se observa más frecuentemente en niños y jóvenes es la DM tipo 1 (DM1) que se puede presentar en edades tempranas y se caracteriza por la destrucción de las células beta del páncreas, lo que se traduce en un déficit absoluto de insulina y dependencia vital de la insulina exógena ${ }^{2}$. Corresponde a más del $80 \%$ de los casos en edad pediátrica, pudiendo presentarse también en adultos jóvenes y representa entre el 5 al 10\% de todos los casos de DM a cualquier edad. $\mathrm{Su}$ mayor incidencia se observa en menores de 15 años, con mayor frecuencia en la edad preescolar y especialmente prepuberal, por lo que también se denominó Diabetes Juvenil o insulinodependiente. Esta se subdivide en Diabetes tipo 1 autoinmune, que corresponde al $90 \%$ de los casos, lo que se evidencia por la presencia de anticuerpos anti-islotes (ICA), antiGAD y anti-insulina y sólo un $10 \%$ de los casos son idiopáticos, catalogados actualmente como: "otras formas de diabetes con severa insulino deficiencia ${ }^{3}$.

En el estudio DiaMond (Diabetes Mondiale Project Group) publicado el año $2000^{4}$, Chile fue clasificado como un país con baja incidencia de DM1 con 2,36 nuevos casos en menores de 15 años por año y cada 100000 habitantes de Santiago (datos obtenidos entre 1990 y 1992). En un estudio más reciente, publicado por Carrasco y colaboradores ${ }^{5}$, se estimó una incidencia de 4,02 nuevos casos en menores de 15 años por año y cada 100.000 habitantes en Santiago. Con estos antecedentes, Chile es actualmente considerado un país de incidencia intermedia de DM1, y esto corrobora la observación de que la incidencia de DM tipo 1 está incrementando en todo el mundo ${ }^{6,7}$.

En los jóvenes también se puede presentar ocasionalmente la DM tipo 2 (DM2), que es la forma más prevalente de la enfermedad y corresponde a una hiperglicemia originada por una resistencia por parte de las células del cuerpo a la acción de la insulina y por un aumento inicial de su secreción por el páncreas; el que finalmente se hace insuficiente para suplir las demandas aumentadas de Insulina. La incidencia de la DM tipo 2 está creciendo en forma alarmante en todo el mundo asociada a la epidemia de obesidad y aunque era considerada una patología de adultos, cada vez es más frecuente en niños y jóvenes y se ha estimado que esto es debido a los hábitos de vida poco saludables en la población ${ }^{8}$.

Las complicaciones médicas más comúnmente asociadas a la DM, incluyen las microangiopáticas como nefropatía, retinopatías, neuropatías, y las macroangiopáticas como enfermedad vascular periférica y enfermedad coronaria $^{9}$. Las complicaciones de la salud oral que se han descrito en asociación a la DM son: 
pérdida de piezas dentarias, gingivitis, periodontitis y patologías de los tejidos blandos ${ }^{1}$.

La gravedad de las complicaciones relacionadas con la salud oral, al igual que las del resto del organismo, tienen directa relación con la hiperglicemia y el tiempo que ha transcurrido desde el inicio de esta. Es por esto que se ha considerado importante conocer los problemas orales en los niños diabéticos para poder efectuar medidas preventivas y oportunas.

\section{Método de Revisión}

Para poder determinar cuáles son las patologías orales que se presentan con mayor frecuencia en los niños diabéticos, su gravedad y complicaciones, se realizó una búsqueda en Internet, ingresando a través del sitio del Sistema de Bibliotecas de la Pontificia Universidad Católica de Chile (SIBUC) a un metabuscador que permite acceder a revistas electrónicas, bases de datos y un catálogo en línea que contiene libros y revistas impresas. Se relacionó el término Salud Oral con Diabetes, Diabetes Mellitus, Diabetes Juvenil y se seleccionaron los trabajos recientes, que realizaron estudios clínicos principalmente en pacientes pediátricos e hicieron comparaciones en cuanto a la salud oral, con niños sanos.

\section{Resultados}

Al incluir en las bases de datos el término diabetes y relacionarlo con salud oral, privilegiando los artículos que habían realizado estudios en niños y jóvenes, se pudo acceder al texto completo de 40 artículos. Al analizarlos, se seleccionaron 12 publicaciones, que habían efectuado estudios clínicos en niños y adolescentes, con una metodología en la que compararon a los afectados por DM con controles sanos y estudiaron diferentes aspectos de su salud oral.

En el trabajo de Evanthia Lalla y cols ${ }^{10}$, se estudió la presencia de caries y enfermedad periodontal en 182 niños y adolescentes diabéticos, entre 6 y 18 años, comparándolos con 160 sujetos semejantes, pero sin la enfer- medad y no hallaron diferencias significativas en la incidencia de caries entre ambos grupos. Ellos encontraron que los niños con DM tenían más placa dental y más inflamación gingival que los controles no diabéticos. La enfermedad periodontal también era significativamente mayor en los niños diabéticos y los resultados del estudio sugerían que la destrucción periodontal puede comenzar muy temprano en la vida del paciente diabético y hacerse más acentuada cuando los niños llegan a la adolescencia. El tener diabetes se correlacionó en forma muy marcada con la presencia de destrucción del tejido conectivo y del hueso alveolar, pero esto según los autores, no dependía de la duración de la diabetes o el nivel de Hemoglobina Glicosilada A 1c (HbA1c). En este estudio el $94 \%$ del total de los niños tenía DM1, y de los chicos mayores (entre 12 y 18 años) un $11 \%$ tenía DM2. Los autores preconizan que al ser la enfermedad periodontal prevenible y la destrucción de los tejidos de soporte dentario controlable, si son detectados precozmente, la revisión periódica de los niños y la implementación de programas preventivos deben ser considerados como rutina en los jóvenes pacientes con diabetes.

En cuanto al control metabólico de la diabetes, éste ha sido relacionado con la salud oral en diferentes estudios. Twetman y cols ${ }^{11}$, estudiaron a 64 niños y adolescentes con DM1, que tenían entre 8 y 15 años de edad. Su principal hallazgo fue que los niños y adolescentes con peor control metabólico, desarrollaron tres veces más lesiones de caries durante el período en estudio, con respecto a aquellos con buen control metabólico, a pesar de los esfuerzos preventivos en salud oral. En otro estudio, el mismo autor encontró una clara correlación entre el riesgo de caries, medido con un programa computacional que determina dicho riesgo y el nivel de control metabólico de la diabetes. Esto lo atribuyeron a factores de comportamiento, ya que un paciente con poco interés en cuidar la dieta, la higiene oral y la salud bucal, podría también tener poca adherencia al tratamiento de la diabetes. Ellos concluyen que hay una relación estadísticamente significativa entre el riesgo de caries y el nivel de HbA1c. También infieren que cuando se 
diagnostica un alto riesgo de caries en un paciente con DM, podríamos tener un pronóstico del nivel de cuidado metabólico que tendrá este mismo paciente en cuanto a la diabetes ${ }^{12}$.

Karjalainen y cols ${ }^{13}$, también investigaron acerca de la caries y su relación con el control metabólico de la diabetes mellitus y concluyeron al estudiar 80 niños y adolescentes con DM1 que un pobre control metabólico de la enfermedad, es un factor de riesgo en cuanto a caries para esos niños y describieron un aumento en el crecimiento de colonias de hongos en la boca de los pacientes con pobre control de la diabetes, lo que podría ser a su vez un indicador de riesgo de caries.

Por otro lado, Siudikiene J y cols ${ }^{14}$, investigaron la relación entre DM1, caries dental y estado salival en niños lituanos. Controlaron a 68 niños y adolescentes entre 10 y 15 años con DM1 comparándolos con 68 controles de similar edad y sexo. A los pacientes con DM1 los dividieron en un grupo con buen a moderado control de la diabetes con $\mathrm{HbA} 1 \mathrm{c}$ menor a 9,0\% y otro grupo con pobre control de la enfermedad con HbA1c mayor a 9,0\%. El grupo de pacientes diabéticos presentó menos caries y placa bacteriana, menos secreción salival, menor efecto buffer de la saliva y mayor frecuencia de candidiasis que los controles no diabéticos. Los pacientes con buen a moderado control de la diabetes tenían menos superficies cariadas en sus dientes, menos recuento de Streptococo Mutans, la bacteria responsable del inicio de la caries dental, y menos hongos que aquellos con pobre control de la diabetes, pero el nivel de control metabólico no tenía influencia en la secreción de la saliva y en su capacidad buffer. Altos niveles de caries en diabéticos fueron significativamente asociados con la edad, cantidad de placa bacteriana y baja producción de saliva, pero no con el nivel de control metabólico de la enfermedad y se producirían por acumulación de placa bacteriana y cambios en la saliva inducidos por la diabetes mellitus.

También se han realizado estudios que relacionan el cuidado de la salud oral con el control de la diabetes, en cuanto al comportamiento de los pacientes y han concluido que de todas las características psicológicas que afectan al progreso de la enfermedad, el desa- rrollo de la conciencia por el autocuidado es el mejor predictor tanto para la salud oral como para la adherencia al cuidado de la diabetes y postulan que incentivando el auto cuidado en una de las áreas se puede mejorar la otra ${ }^{15}$. La educación en salud, promoviendo un buen cuidado dental, podría tener un efecto benéfico en la adherencia al control de la diabetes ${ }^{16}$.

En el trabajo de Ali-Asghar Alavi y cols, realizado en Irán, en la Universidad de Ciencias Médicas de Shiraz, estudiaron cincuenta pacientes (22 niños y 28 niñas) con DM1 metabólicamente bien controlada y les midieron el índice COPD (dientes cariados, obturados y perdidos) con radiografías y con examen clínico. Los resultados fueron comparados con los obtenidos en un grupo de escolares de control. El índice COPD en los pacientes diabéticos fue más alto que en el grupo control, lo que atribuyen a la reducción de secreción salival en los enfermos diabéticos, poco cuidado con la dieta y falta de higiene oral (un $44 \%$ de los pacientes en este estudio no cepillaban sus dientes). Los primeros molares permanentes inferiores fueron los que con más frecuencia presentaban caries y se estableció una relación directa entre el estado de salud del primer molar permanente y el tener DM1 ${ }^{17}$. Para reducir las caries dentales en los pacientes diabéticos, los autores aconsejan visitas regulares al dentista, la implementación de medidas preventivas como terapia con flúor, uso de sellantes, control de la glicemia y prevención de la xerostomía.

En Turquía, Recep Orbak y cols ${ }^{18}$, controlaron a 50 niños con DM1 y los compararon con otros 50 no diabéticos. Ellos querían investigar la influencia de la diabetes mellitus tipo 1 en la dentición y en la salud oral. Los niños tenían entre 5 y 14 años. En su estudio, la inflamación gingival que acompaña la erupción dentaria era mayor en los individuos diabéticos que en los no diabéticos. También observaron, en los niños con DM1, una aceleración en la dentición hasta la edad de 10 años y un retardo posteriormente, esto lo atribuyen a que enfermedades como la diabetes, producen una inestabilidad metabólica. El intervalo edéntulo era más largo en el grupo con diabetes y esto se acompañaba con un mayor índice de inflamación gingival. Sus resultados mostraron que 
había más placa bacteriana y más depósitos de cálculos en los niños diabéticos que en el grupo control. En relación a las caries, sus resultados revelaron que los niños con DM1 tenían menos caries que los controles no diabéticos a todas las edades.

En el trabajo de Shantanu Lal y cols ${ }^{19}$, también se estudió la influencia de la diabetes mellitus en la erupción dentaria. Ellos compararon 270 niños con diabetes con 320 niños control, en la edad de dentición mixta, desde los 6 a los 14 años. Sus resultados indicaron que los niños diabéticos tenían una erupción dentaria acelerada, la que era más pronunciada a medida que aumentaba la edad. No hubo diferencias en la exfoliación de las piezas temporales. Según los autores, la respuesta exagerada a la placa bacteriana, típica de la enfermedad periodontal asociada a la hiperglicemia, podría contribuir a que se produjeran cambios óseos localizados, con el resultado de disminuir la calidad y cantidad del hueso que rodea las piezas dentarias, lo que según los autores aceleraría la erupción.

Evanthia Lalla y cols ${ }^{20}$, en un extenso estudio, compararon a 350 niños con diabetes mellitus, con edades entre 6 y 18 años, reclutados en el Centro para Diabéticos Naomi Berrie de la Universidad de Columbia, con 350 niños no diabéticos, en cuanto a los signos de cantidad de placa bacteriana, inflamación y sangramiento gingival y pérdida de la inserción epitelial (síntoma de enfermedad periodontal) encontrando aumentos significativos de la enfermedad gingival y periodontal en los niños con diabetes, comparados con los de control. Incluso al dividir a los niños estudiados en menores de 12 años y mayores de esa edad, los síntomas de enfermedad periodontal eran significativamente mayores en los niños diabéticos de ambos grupos. Esto demostraría una asociación entre la diabetes y un aumento del riesgo de padecer enfermedad periodontal, incluso en etapas tempranas de la vida.

En un siguiente trabajo, los mismos autores $^{20}$, relacionaron tanto el sangramiento gingival cómo la pérdida de inserción epitelial y su estudio reveló que el nivel de $\mathrm{HbAlc}$ tiene una relación significativa con la periodontitis. Estos resultados sugieren que hay una destrucción periodontal acelerada en las personas jóvenes con diabetes, en relación al nivel de control metabólico. Un buen control metabólico sería importante para prevenir las complicaciones en jóvenes con diabetes, similar a lo que está bien establecido para otras complicaciones sistémicas de la enfermedad.

Los resultados de estos estudios nos señalan a la enfermedad periodontal como una de las primeras complicaciones de la diabetes mellitus ya que los niños y adolescentes estudiados, no presentaban evidencia de otra complicación y demuestran la relación con el control metabólico de la diabetes.

Es por esto que autores como Moore, Guggenheimer y Weyant ${ }^{1}$, que estudiaron en su trabajo a 406 individuos adultos con DM tipo 1, y los compararon con 203 sujetos de control, en cuanto a sus hábitos de cuidado oral, acceso a la atención dental y necesidad de mejorar su educación en salud, realzan el papel que los dentistas tienen y su responsabilidad en promover buenos hábitos de salud oral, controlar periódicamente a sus pacientes y desaconsejar el uso del tabaco, lo que puede mejorar significativamente la salud oral de los pacientes diabéticos.

\section{Discusión}

Según la literatura consultada, la salud oral de los niños y jóvenes se ve afectada por el hecho de padecer diabetes. Los múltiples estudios que han buscado la relación entre la caries y la diabetes mellitus, no nos entregan un resultado unánime, algunos simplemente descartan esa relación, argumentando que la dieta pobre en carbohidratos de los niños diabéticos los protege de las caries.

Para otros autores habría un aumento de las caries en los pacientes diabéticos, especialmente en los que tienen un pobre control metabólico de la enfermedad. Esto se explica por factores como la disminución del flujo salival que presentan los pacientes diabéticos, el aumento de la glucosa en la saliva, la disminución del efecto tampón de la misma y malos hábitos de autocuidado en general.

Otro efecto de la diabetes en los niños tendría relación con un aceleramiento en el re- 
cambio dentario, esto es importante porque las piezas recién erupcionadas son más susceptibles a cariarse y mientras menor es el niño, sus hábitos de higiene oral pueden estar menos arraigados y esto favorecer la formación de caries.

En cuanto a la enfermedad gingival y periodontal, todos los autores están de acuerdo en que se presenta con mayor prevalencia y severidad en los niños con diabetes y aparece en forma precoz. Si tenemos en cuenta que ya en 1993, Löe ${ }^{22}$, determinó que la enfermedad periodontal era la sexta complicación de la diabetes mellitus y en 2008 Taylory Borgnakke ${ }^{23}$, establecieron que la enfermedad periodontal es un posible factor de riesgo para un buen control metabólico de la diabetes, no cabe duda de la importancia en la prevención y cuidado de los tejidos periodontales en los niños, donde se pueden realizar precozmente labores de educación y formación de hábitos.

\section{Conclusiones}

La DM es una enfermedad metabólica, crónica e irreversible que afecta la salud de diferentes órganos y sistemas de la persona que la padece. Cada vez hay una mayor incidencia de enfermos diabéticos en todo el mundo y de edades más precoces. La salud oral de los pacientes diabéticos también está alterada por su condición y en niños ya se pueden observar complicaciones atribuibles a su enfermedad. El pobre control metabólico de la diabetes afecta negativamente a la salud oral de los pacientes, pudiéndose observar mayor riesgo de caries. Para reducir la incidencia de caries en los pacientes diabéticos, los estudios recomiendan que se deben implementar las medidas de cuidado oral habituales en la infancia, en forma rigurosa, con visitas regulares al dentista, acciones preventivas como terapias con fluoruros, uso de sellantes, buen cuidado de la dieta, control de la glicemia, control y prevención de la xerostomía y una educación en salud oral efectiva y continua.

Los tejidos periodontales se comprometen en forma severa y precoz en los niños diabéticos por lo que la progresión de su destrucción puede ser evitada cuando se identifica en sus primeros estados de enfermedad y se implementan factores de prevención y tratamiento oportunos.

La educación en salud, evitando hábitos como el tabaquismo y sedentarismo, fomentando una dieta saludable, inculcando hábitos de higiene oral y de control metabólico de la diabetes, deben iniciarse precozmente y el odontólogo debe participar en el equipo tratante de los niños y jóvenes diabéticos para aportar desde su ámbito a la prevención de serias complicaciones.

De la misma manera, es fundamental, que se considere la inclusión del control odontológico para los niños y jóvenes con diabetes mellitus, en las guías clínicas ministeriales, que entregan las pautas para la atención de estos pacientes.

\section{Referencias}

1.- Moore PA, Guggenheimer TOJ, Weyant RJ: Diabetes and Oral Health Promotion: a survey of disease prevention behaviors, J Am Dent Assoc 2000; 131: 1333-41.

2.- Kawasaki E, Abiru N, Eguchi K: Prevention of type 1 diabetes: from the view point of $\beta$ cell damage. Diab Res Clin Pract 2004; 66 (Suppl 1): 27-32.

3.- Ministerio de Salud: Guía Clínica Diabetes Mellitus Tipo 1. 1st Ed. Santiago: Minsal, 2005.

4.- Karvonen M, Viik-Kajander M, Moltchanova E, Libman I, LaPorte R, Tuomilehto J: Incidence of childhood type 1 diabetes worldwide. Diabetes Mondiale Project Group (DiaMond). Diabetes Care 2000; 23: 1516-26.

5.- Carrasco E, Pérez-Bravo F, Dorman J, Mondragón A, Santos JL: Increasing incidence of type 1 diabetes in population from Santiago of Chile: trends in a period of 18 years (1986-2003). Diabetes Metab Res Rev 2006; 22: 34-7.

6.- Gale E: A. The rise of childhood type 1 diabetes in the 20th century. Diabetes 2002; 51: 3353-61.

7.- Eyzaguirre F, Pelaez JM, Sepúlveda C, et al: Diabetes Mellitus tipo 1 (DM1) en niños menores de 5 años: Características al debut vs otros grupos etarios en Chile. Rev Chil Pediatr 2006; 77: 375-81.

8.- Alberti G, Zimmet P, Shaw J, Bloomgarden Z, et $a l$ : Type 2 Diabetes in the Young: The Evolving Epidemic: The International Diabetes Federation Consensus Workshop. Diabetes Care 2004; 27: 1798-811.

9.- Orchard TJ, Dorman JS, Maser RE, et al: Prevalence of complications in IDDM by sex and duration. Pittsburgh 
Epidemiology of Diabetes Complications Study II. Diabetes 1990; 39: 1116-24.

10.- Lalla E, Cheng B, Lal S, Tucker S, et al: Periodontal Changes in Children and Adolescents With Diabetes: A case-control study. Diabetes Care 2006; 29: 295-9.

11.- Twetman S, Johansson I, Birkhed D, Nederfors T: Caries incidence in young Type 1 diabetes mellitus patients in relation to metabolic control and caries-associated risk factors. Caries Research 2002; 36: 31-5.

12.- Twetman S, Petersson GH, Bratthall D: Caries risk assessment as a predictor of metabolic control in young Type 1 diabetics. Diabetes UK. Diabetic Medicine 2005; 22: 312-5.

13.- Karjalainen KM, Knuuttila MLE, Käär ML: Relationship between caries and level of metabolic balance in children and adolescents with insulin-dependent diabetes mellitus. Caries Research 1997; 31: 13-8.

14.- Siudikiene J, Machiulskiene V, Nyvad B, Tenovuo J, Nedzelskiene I: Dental caries and salivary status in children with type 1 diabetes mellitus, related to the metabolic control of the disease. Eur J Oral Sci 2006; 114: 8-14.

15.- Syrjälä A-MH, Ylöstalo P, Niskanen MC, Knuuttila $M L E$ : Relation of different measures of psychological characteristics to oral health habits, diabetes adherence and related clinical variables among diabetic patients. Eur J Oral Sci 2004; 112: 109-14.
16.- Syrjälä AMH, Niskanen MC, Knuuttila MLE: The theory of reasoned action in describing tooth brushing, dental caries and diabetes adherence among diabetic patients. J Clin Periodontol 2002; 29: 427-32.

17.- Alavi AA, Amirhakimi E, Karami B: The prevalence of dental caries in 5-18-year-old insulin-dependent diabetics of Fars Province, Southern Iran. Arch Iran Med 2006; 9: 254-60.

18.- Orbak R, Simsek S, Orbak Z, Kavrut F, Colak M: The Influence of Type-1 Diabetes Mellitus on Dentition and Oral Health in Children and Adolescents. Yonsei Med J 2008 49: 357-65.

19.- Lal S, Cheng B, Kaplan S, et al: Accelerated Tooth Eruption in Children with Diabetes Mellitus. Pediatrics 2008; 121: 1139-43.

20.- Lalla E, Cheng B, Lal S, et al: Diabetes mellitus promotes periodontal destruction in children. J Clin Periodontol 2007; 34: 294-8.

21.- Lalla E, Cheng B, Lal S, et al: Diabetes-related parameters and periodontal conditions in children. J Periodont Res 2007; 42: 345-9.

22.- Löe H: Periodontal disease: The sixth complication of diabetes mellitus. Diabetes Care 1993; 16: 329-34.

23.- Taylor GW, Borgnakke WS: Periodontal disease: associations with diabetes, glycemic control and complications. Oral Diseases 2008, 14: 191-203. 